

\title{
WestminsterResearch
}

http://www.wmin.ac.uk/westminsterresearch

\section{Novel SRR loaded waveguide bandstop filters.}

\section{Alexander B. Shelkovnikov}

N. Suntheralingam

Djuradj Budimir

School of Informatics, University of Westminster

Copyright (C) [2006] IEEE. Reprinted from the proceedings of the IEEE Antennas and Propagation Society International Symposium 2006, pp. 45234526.

This material is posted here with permission of the IEEE. Such permission of the IEEE does not in any way imply IEEE endorsement of any of the University of Westminster's products or services. Internal or personal use of this material is permitted. However, permission to reprint/republish this material for advertising or promotional purposes or for creating new collective works for resale or redistribution must be obtained from the IEEE by writing to pubs-permissions@ieee.org. By choosing to view this document, you agree to all provisions of the copyright laws protecting it.

The WestminsterResearch online digital archive at the University of Westminster aims to make the research output of the University available to a wider audience. Copyright and Moral Rights remain with the authors and/or copyright owners.

Users are permitted to download and/or print one copy for non-commercial private study or research. Further distribution and any use of material from within this archive for profit-making enterprises or for commercial gain is strictly forbidden.

Whilst further distribution of specific materials from within this archive is forbidden, you may freely distribute the URL of the University of Westminster Eprints (http://www.wmin.ac.uk/westminsterresearch).

In case of abuse or copyright appearing without permission e-mail wattsn@wmin.ac.uk. 


\title{
Novel SRR Loaded Waveguide Bandstop Filters
}

\author{
A. Shelkovnikov, N. Suntheralingam, and D. Budimir \\ Wireless Communications Research Group, Department of Electronic Systems, \\ University of Westminster, London, W1W 6UW, UK
}

\section{Introduction}

The explosive growth in commercial interest in RF, microwave and millimeterwave systems, especially in wireless communications and mobile and satellite communication systems has provided a significant challenge to conventional microwave circuits and their design methodologies. High performance narrowband bandpass filters having a low insertion loss, compact size, wide stopband and a high selectivity are important for next-generation wireless systems. At present most filters at RF, microwave and millimeter-wave frequencies are produced either in waveguide (air-filled metal pipe, dielectric-filled or micromachined air-filled) with high associated machining costs, image guide and nonradiative dielectric guide with high associated loss or using dielectric resonators or using printed circuit board (PCB) and multi-layered lowtemperature-cofired-ceramics (LTCC) technologies.

Rectangular waveguides have been a sustainable solution over the past few decades, used to design robust, low loss and high power circuits at microwave and millimeter-wave frequencies. The classical rectangular waveguide theory is still very much usable in order to build various filter structures, which are viable to meet requirements of the modern technology [1]. However, reduction of the physical size of such structures has become one of the primary goals. Recently proposed concepts of left-handed medium (LHM) have become the subject of extensive investigations due their capability to provide novel unconventional properties to different propagation media [2]-[4]. This approach makes use of the left-handed medium created by a novel type of resonance elements, split ring resonators (SRRs), in combination with the thin metal wireline [4]. These are printed on the dielectric slab, which is then inserted into the plane of symmetry of the rectangular waveguide. These structures are able to alter the electromagnetic boundary conditions of the surface and prohibit propagation of signal in a certain frequency band. Thus, the traditional miniaturization techniques, which commonly employ dielectric-filled waveguides with standard dimensions bound to the wavelength $(\lambda)$, may be enhanced to achieve more compact highperformance waveguide components.

In this paper, we make use of the left-handed properties imposed by SRRs in order to achieve miniaturization of rectangular waveguide filters. Analysis and design of the SRR-loaded and multilayer bandstop filters are presented. Simulated and experimental results demonstrate feasibility of the proposed structures. 


\section{SRR-Loaded Waveguide Bandstop Filter}

The SRR-loaded waveguide bandstop filter is realized as a cascade of the resonator unit cells. The transmission line is loaded with the slab of a composite material, which conveniently facilitates both split ring resonators with the metal septa on the top plane, and a thin wireline stretched throughout the full length of the dielectric on its bottom plane. Configuration of the SRR-loaded waveguide bandstop filter is shown in Fig. 1.

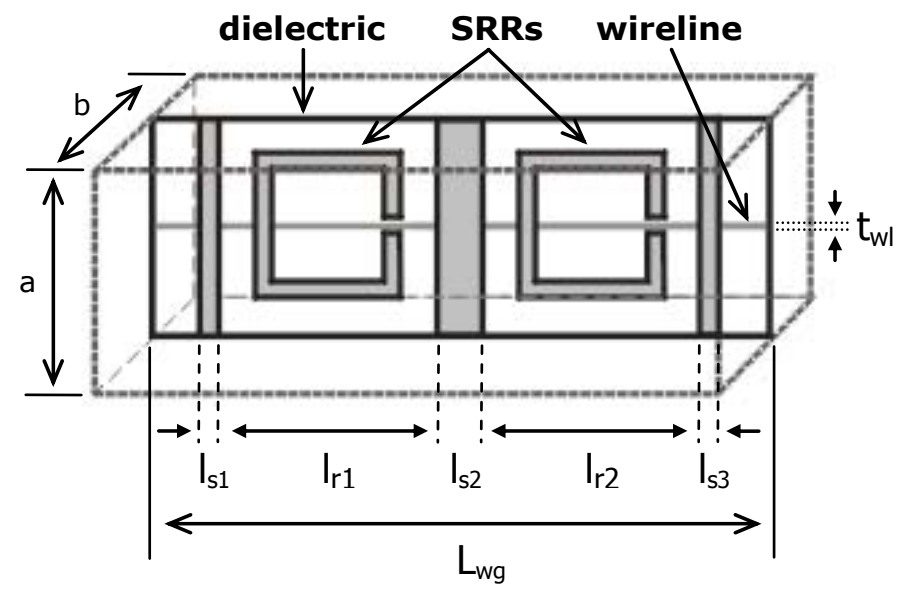

Fig. 1. Configuration of an SRR-loaded waveguide bandstop filter

\begin{tabular}{|l|c|}
\hline Parameter & (in mm) \\
\hline Thickness of the wireline, $t_{w 1}$ & 0.2 \\
\hline Length of metal septa, $I_{s 1}=I_{s 3}$ & 0.2 \\
\hline Length of metal septum, $I_{s 2}$ & 1.0 \\
\hline Length of resonator sections, $I_{r 1}=I_{t 2}$ & 8.0 \\
\hline Total length of the waveguide filter, $L_{w a}$ & 17.4 \\
\hline
\end{tabular}

Table I. Dimensions of the SRR-loaded bandstop filter

Split ring resonators are characterized by the following parameters: ring thickness $(s=0.25 \mathrm{~mm})$, inner diameter $\left(w_{0}=2.62 \mathrm{~mm}\right)$ and ring gap $(g=0.48 \mathrm{~mm})$. The filter was designed and simulated with its dimensions presented in Table I. In order to illustrate the feasibility of the proposed structure the SRR-loaded waveguide bandstop filter has been fabricated and tested at X-band. Standard rectangular waveguide (WG-16: $a=22.86 \mathrm{~mm}, b=10.16 \mathrm{~mm}$ ) has been used as a housing to fit a $0.508-\mathrm{mm}$-thick dielectric slab. As illustrated in Fig. 2, the filter performs a wide stopband of $1 \mathrm{GHz}$, which can be controlled by tuning the resonance of SRRs and lengths of metal discontinuities. The measured response demonstrates agreement with the simulation. 


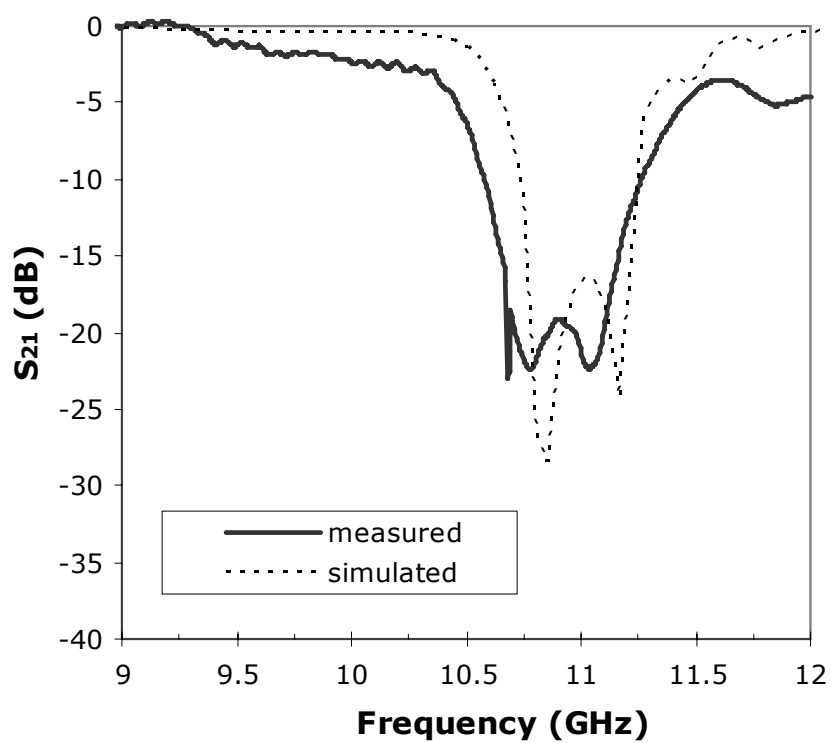

Fig. 2. Simulated and measured insertion loss of the SRR-loaded waveguide bandstop filter

\section{Multilayer SRR-Loaded Waveguide Bandstop Filter}

The proposed bandstop filter structure is shown in Fig. 3. The structure consists of a two block of dielectrics, which are transversely inserted inside a rectangular waveguide. Two transverse periodic SRRs are present on either side of the dielectric blocks. The dielectrics, which have $\varepsilon_{r}=2.2$ and thickness $h=0.254 \mathrm{~mm}$, support these SRRs.

The space between the dielectric blocks $(L=2.254 \mathrm{~mm})$ and input and output of the waveguide is filled with air. Standard rectangular waveguide WG-16 has been used as housing. Fig. 4 shows the S-parameters response for multilayer SRRloaded waveguide bandstop filter at $11 \mathrm{GHz}$.

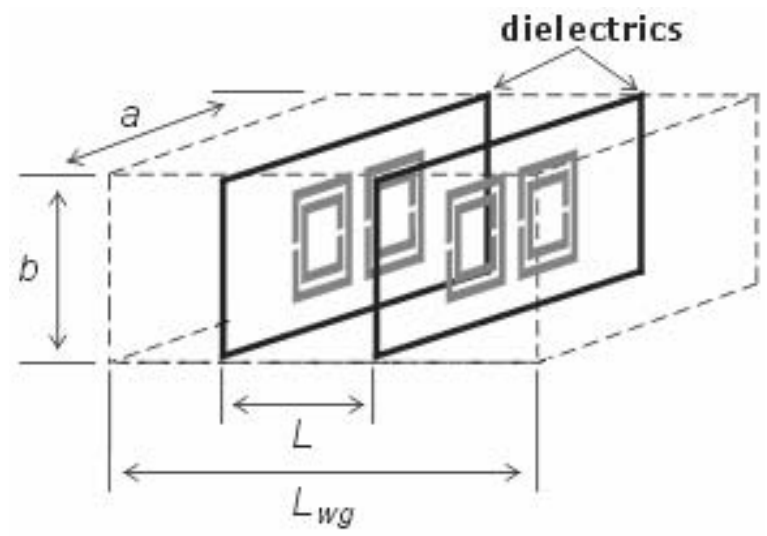

Fig. 3. Configuration of the proposed multilayer waveguide bandstop filter together with the waveguide housing 


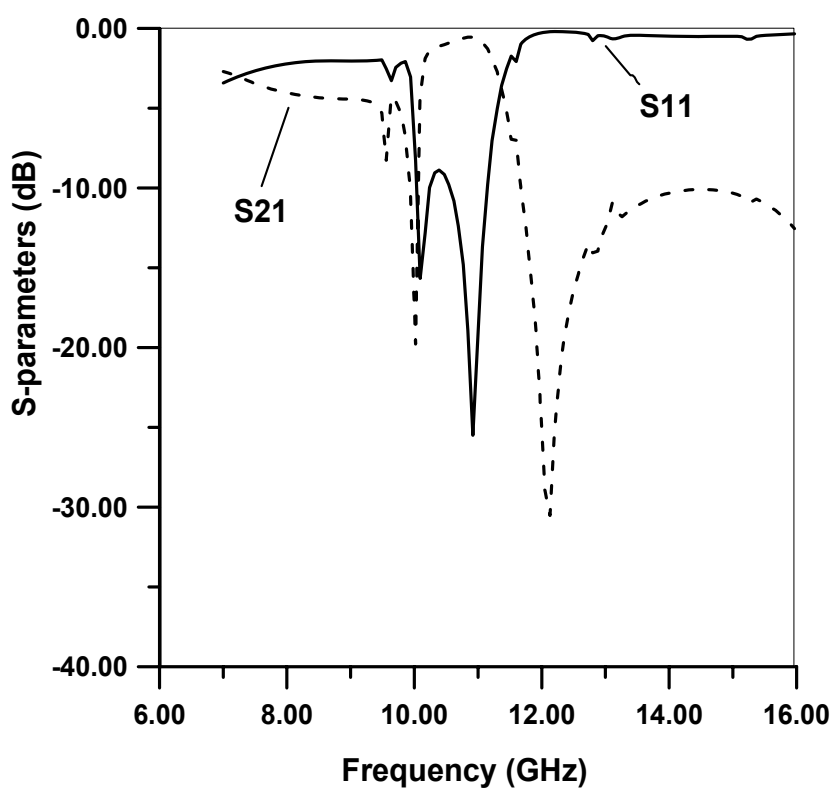

Fig. 4. Simulated S-parameters of the multilayer waveguide bandstop filter

\section{Conclusions}

Two types of novel split ring resonator (SRR) loaded rectangular waveguide bandstop filter structures have been presented. The first proposed SRR-loaded waveguide bandstop filter has been designed simulated and tested. Using the combination of the split ring resonators, conductive wireline and transversely placed metal septa, the composite material has been built and inserted into the hollow rectangular waveguide to form an SRR-loaded waveguide unit. The bandstop filter has been constructed by cascade of the two resonator unit cells. The second proposed multilayer SRR loaded waveguide bandstop filter has been designed and simulated. Three transverse periodic SRRs are present on either side of the dielectric blocks.

\section{References:}

[1] V. Postoyalko, and D. Budimir, "Design of Waveguide E-plane Filters with All-Metal Inserts by Equal-Ripple Optimization," IEEE Trans. Microwave Theory \& Tech., vol. MTT-42, pp. 217-222, February 1994

[2] V. G. Veselago, "The electrodynamics of substances with simultaneously negative values of $\varepsilon$ and $\mu$," Soviet Phys. Uspekhi, vol. 10, no. 4, pp. 509-514, Jan.-Feb. 1968

[3] J.B. Pendry, A.J. Holden, D.J. Robbins, and W.J. Stewart, "Magnetism from conductors and enhanced nonlinear phenomena," IEEE Trans. Microwave Theory \& Tech., vol. 47, no. 11, pp. 2075-2084, November 1999

[4] D. R. Smith, W. J. Padilla, D. C. Vier, S. C. Nemat-Nasser, and S. Schultz, "Composite medium with simultaneously negative permeability and permittivity," Phys. Rev. Lett., vol. 84, pp. 4184-4187, May 2000

[5] Ansoft HFSS, v.9.0, 2004 